\title{
Analysis of various properties of polyvinyl alcohol-graphene oxide nanocomposite films
}

\author{
Nitish Raja*, Dhiraj Kumar Rana and Soumen Basu \\ Department of Physics, National Institute of Technology, Durgapur-713209, India

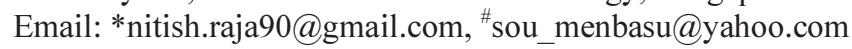

The pure phase Graphene oxide (GO) was prepared by hummers method and was confirmed by XRD analysis. In XRD pattern a sharp peak appeared at angle $2 \theta=11.01^{\circ}$ (Figure 1a) PVA-GO nanocomposite with varying $\mathrm{GO} \mathrm{wt}^{\mathrm{t}} \%(0,2$ and $8 \mathrm{wt} \%)$ was synthesized by drop casting method and characterized by help of various characterization techniques. Surface morphology of composite films is studied by SEM analysis (Figure 1b-1d). Thermal behavior of PVA-GO nanocomposites with different weight $\%$ is analyzed by DTA-TGA curve (Figure 2).

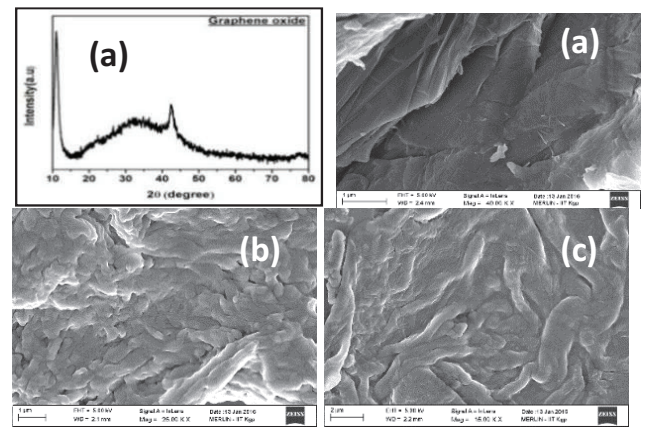

Figure 1: (a) XRD pattern of pure Graphene oxide. (b-d) SEM images of (b) PVA, (c) PVA-GO (2wt \%) and (d) PVA-GO ( $8 \mathrm{wt} \%$ )

From DTA analysis it is observed that the peak obtained at around $230{ }^{\circ} \mathrm{C}$ for PVA and PVAGO nanocomposites represents the onset of degradation and it also confirmed from TGA analysis. TGA analysis showed the huge weight loss nearly $82 \%$ obtained for PVA, PVA-GO nanocomposites and for pure GO the weight loss is nearly $62 \%$ in temperature range 230 $370^{\circ} \mathrm{C}$. The thermal stability of GO increased with increasing GO weight \% in PVA-GO nanocomposites. The temperature dependent electrical properties such as resistivity ( $\rho)$, dielectric constant $(\varepsilon)$, tangent loss $(\tan \delta)$ of PVA-GO nanocomposites are investigated at temperature range $30<\mathrm{t}<150{ }^{\circ} \mathrm{C}$ (Figure 3). The temperature dependent dielectric constant plot showed that $\varepsilon$ increased from 18.9 to 34.5 with increasing GO wt \% in PVA, PVA-GO $(2 \mathrm{wt} \%)$ respectively. At room temperature the dielectric constant is increased from 18.9 to 34.5 and resistivity decreased from 22.01 to 9.5 $\Omega \mathrm{m}$ with increasing $\mathrm{GO} \mathrm{wt} \%$ in PVA-GO composites. Arrhenius relation (eq. 1) is used for determining the activation energy of the samples. The tangent loss $(\delta)$ obtained for PVA, PVA-GO nanocomposite is approximately 0.2 .

$$
\rho(T)=\rho(0) \exp \left[\frac{E_{a}}{K_{\beta} T}\right]
$$

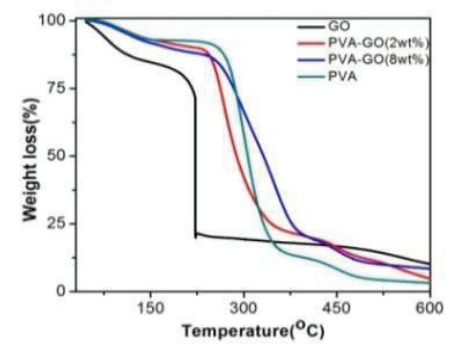

Figure 2: Temperature dependent weight loss of PVA-GO nanocomposite

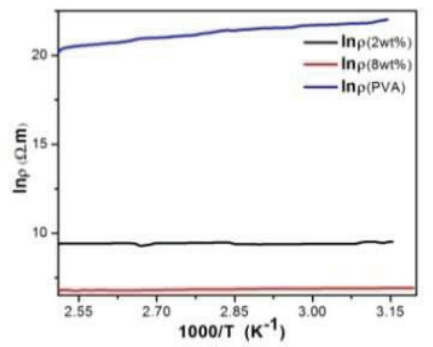

Figure 3: Temperature dependent resistivity of PVA-GO nanocomposite

\section{References}

1. Zhu et.al, "Graphene and Graphene Oxide: Synthesis, Properties, and Applications", Advanced materials XX, (2010) 1-19.

2. Park et.al, "Preparation of novel CdSgraphene/ $/ \mathrm{TiO}_{2}$ composites with high photocatalytic activity for methylene blue dye under visible light", Bulletin of Materials Science, 36, no. 5 (2013) 869876. 